\title{
Анализ гибридных регуляторов в моделях управления техническими объектами в изменяющихся условиях
}

\author{
B.В. Игнатьев ${ }^{1}$, к.m.н., ведущий научный сотрудник, vvignatev@sfedu.ru \\ В.В. Соловьев ${ }^{1}$, ст. преподаватель \\ Д.А. Белоглазов ${ }^{1}$, к.т.н., доцент
}

${ }_{1}^{1}$ Южный федеральный университет, г. Таганрог, 347922, Россия

\begin{abstract}
В статье рассмотрены модели, управление в которых осуществляется с помощью гибридных регуляторов, реализованных на основе последовательного взаимодействия PI- и IPI-FUZZY-регуляторов, а также PID- и IPD-FUZZY-регуляторов со сгенерированной структурой системы нечеткого вывода типа Сугено и разработанной моделью ANFIS.

В гибридных регуляторах база правил нечеткого регулятора формируется автоматически с помощью специально разработанного алгоритма на основе данных, полученных с классического регулятора с последующим обучением с помощью нейросети. Особенностью разработки ANFIS в виде гибридной сети для PI- и IPI-FUZZY-регуляторов является использование показателей ошибки выходного сигнала, ее интеграла (дифференциала для PID- и IPD-FUZZY-регуляторов) и управляющего воздействия. Для проверки эффективности гибридной сети с целью выявления факта ее переобучения применялись данные, полученные в результате работы классического регулятора, а для формирования обучающей выборки для построения гибридной сети - данные, полученные в результате работы нечеткого регулятора. Это позволяет исключить участие эксперта при синтезе базы правил нечеткого регулятора и обеспечить эффективное и робастное управление объектом, функционирующим в непредвиденных внешних ситуациях.

Регуляторы IPI-FUZZY и IPD-FUZZY продемонстрировали лучшие показатели качества по сравнению с соответствующими классическими регуляторами, что позволяет рекомендовать их к применению в реальных системах управления. Представленные модели были разработаны в среде Simulink и редакторе ANFIS пакета расширения Fuzzy Logic Toolbox.

Ключевые слова: управление, гибридная модель, интеллектуальный регулятор, база правил, обучение, неопределенность.
\end{abstract}

Автоматизация производства определяет эффективность технологических процессов, построенных на основе интеграции разных подсистем, к которым предъявляются требования безотказной совместной работы. Поэтому АСУ технологическими процессами (АСУ ТП) являются основой любого производства. От эффективности управления отдельными объектами с помощью АСУ ТП зависит эффективность управления производством в целом.

Стремительное развитие современных АСУ ТП определяется необходимостью решать сложные задачи, связанные с обеспечением надежного управления в условиях неопределенности. Для решения таких задач применяются интеллектуальные технологии, позволяющие обеспечить надежность и оперативность управления, в том числе в нештатных ситуациях.

Однако практически во всех существующих АСУ ТП в контурах управления отдается предпочтение традиционным методам управ- ления, основанным на применении классических регуляторов, несмотря на их ограниченные возможности или неприменимость для управления техническими объектами со слабоформализованной структурой, динамическим поведением, в случае изменения параметров объекта, при функционировании в непредвиденных внешних ситуациях.

Это обусловлено определенной надежностью классических регуляторов при управлении стационарными объектами и недоверием к интеллектуальным из-за их ограниченной применимости на реальных производствах, в том числе современных, и отсутствия опыта эксплуатации.

Для внедрения интеллектуальных систем управления на реальных производствах необходимо получить достаточно теоретических и прикладных доказательств их надежности, способности гарантировать получение желаемого управления с максимальным качеством в случае изменения ситуаций управления. 
Данная работа посвящена анализу результатов проведенных авторами исследований по разработке систем управления техническими объектами, функционирующих в условиях неопределенности на основе интеллектуальных гибридных регуляторов - классических (PI, PID) и нечетких (IPI-FUZZY, IPD-FUZZY) и позволяющих обеспечить желаемое управление с максимальным качеством, в том числе в случаях изменения ситуаций управления.

Ставится задача анализа сигналов управления, получаемых в гибридных регуляторах на основе сравнения результатов работы классического PI-регулятора с IPI-FUZZYрегулятором и классического PID-регулятора с IPD-FUZZY-регулятором.

\section{Анализ исследований по научной проблеме}

Проблеме разработки систем управления с применением интеллектуальных регуляторов мировым научным сообществом уделяется особое внимание и посвящено немало научных работ.

Так, в исследовании [1] рассмотрены правила настройки для надежных контроллеров FOPID на основе многоцелевой оптимизации с использованием моделей FOPDT. Предложен набор оптимально сбалансированных правил настройки для ПИД-регуляторов дробного порядка с минимизацией интегрированной абсолютной ошибки как для заданного значения, так и для отклика на возмущение нагрузки.

Работа [2] посвящена разработке гибридной системы, в которой оптимальность параметров ПИД-регулятора, полученных с использованием генетического алгоритма, достигается посредством нейронной сети, а затем также с ее помощью строится база правил нечеткой модели.

Разработка нечеткого регулятора на основе улучшенного алгоритма генетической оптимизации для управления беспилотным вертолетом обсуждается в [3]. Улучшенный авторами генетический алгоритм используется для оптимизации исходных экспертных эмпирических правил нечеткости, что позволяет избежать применения традиционных методов при локальных решениях в процессе оптимизации. Результаты моделирования синтезированного адаптивного нечеткого ПИД-регулятора показали, что он обладает лучшими управляющим эффектом и защитой от помех, чем классический ПИД-регулятор. Однако разработанный генетический алгоритм используется для оптимизации исходных экспертных эмпирических правил нечеткости, изначально зависящих от знаний экспертов, что не является преимуществом перед системами управления, в которых правила нечеткого вывода генерируются автоматически.

Логика на основе гибридного управления для нечеткого ПИД-регулирования представлена в работе [4]. Настройка параметров нечеткого регулятора происходит в реальном времени на основе алгоритма Мамдани с учетом идентификации, выполняемой при влиянии на поведение процесса управления неизмеряемого ступенчатого возмущения любой формы.

В работе [5] описан эффективный метод определения оптимальных параметров для настройки нечеткого ПИД-регулятора. Недостатком метода является отсутствие обучения или адаптации регулятора в случае изменения параметров объекта управления.

Реализации гибридного подхода к задаче управления техническим объектом на основе классического и нечеткого ПИД-регуляторов для настройки мощности активной зоны жидкосолевого реактора посвящена статья [6]. Чтобы одновременно использовать преимущества нечеткого и традиционного ПИДрегуляторов, разработан комбинированный регулятор, который автоматически переключается между нечетким управлением и ПИДуправлением в соответствии с диапазоном ошибок. В результате моделирования определено, что показатели переходных процессов комбинированного регулятора выше, чем у обычного ПИД-регулятора или нечеткого регулятора.

В работе [7] синтезирован нечеткий регулятор на основе традиционного ПИД-регулятора. Данные замкнутого контура, включая сигналы ошибок и управления, анализируются для получения наиболее подходящих входных и выходных лингвистических переменных и базы правил нечеткого регулятора, которые описывают поведение, а точнее динамику системы. Предлагаемый метод определяет основные параметры нечеткого регулятора и помогает избежать сложностей, связанных с представлением экспертных знаний. Далее разрабатывается нечеткий регулятор, который должен поддерживать производи- 
тельность традиционного ПИД-регулятора и быть невосприимчивым к неопределенности коэффициентов модели.

Система управления асинхронным приводом переменного тока на основе адаптивного нечеткого идентификатора и нейронечеткого регулятора представлена в работе [8]. Система способна адаптироваться к изменениям нагрузки линии электропередачи, меняя правила нейронечеткого регулятора.

Авторами работы [9] предложен генетический подход для автоматической настройки адаптивного нечеткого ПИД-регулятора системы слежения телескопа. Разработан нечеткий адаптивный ПИД-регулятор положения сервопривода оси телескопа. Представлена автоматическая настройка функций принадлежности и правил нечеткого регулятора с применением эволюционного алгоритма. Данный регулятор эффективен при высокоточном управлении за счет исключения ступенчатого режима, возникающего в многомассовой системе с нелинейным крутящим моментом при очень низкой скорости слежения за космическими объектами.

Анализ научных исследований в области проектирования интеллектуальных систем управления, автоматических процедур их синтеза позволяет сделать вывод, что одними из основных направлений развития гибридных систем являются комбинации классического и нечеткого регуляторов, применение нейросети, генетических алгоритмов.

В данной работе рассматриваются гибридные регуляторы, построенные на интеграции классического и нечеткого регуляторов с применением нейросети, что определяет актуальность проводимых исследований.

\section{Метод решения задачи управления техническими объектами в условиях неопределенности}

Данная работа является продолжением проведенных авторами исследований по разработке методов эффективного управления на основе гибридного подхода [10], интеллектуальных регуляторов $[11,12]$ и методов их оптимизации [13].

Метод, позволяющий обеспечить желаемое управление техническим объектом, функционирующим в изменяющихся условиях, определен следующими шагами.

1. Разработка гибридной модели управления техническими объектами с применением
PI- и IPI-FUZZY-регуляторов, PID- и IPDFUZZY-регуляторов. В гибридных моделях базы правил для нечетких регуляторов формируются автоматически с помощью разработанного алгоритма [14] на основе эталонных систем, под которыми понимаются контуры управления с помощью классических PI- и PID-регуляторов соответственно. Далее выполняется запуск моделей для сохранения показателей работы классического и нечеткого регуляторов (показатели записываются в специальные программные файлы).

2. Разработка модели ANFIS в виде гибридной сети на основе PI- и IPI-FUZZYрегуляторов, а также PID- и IPD-FUZZY-peгуляторов.

При реализации модели на основе PI- и IPI-FUZZY-регуляторов показатели ошибки выходного сигнала, ее интеграла и управляющего воздействия, полученные в результате работы классического регулятора, являются данными, используемыми для проверки эффективности гибридной сети с целью выявления факта ее переобучения, а показатели ошибки выходного сигнала, ее интеграла и управляющего воздействия, полученные в результате работы нечеткого регулятора, формируют обучающую выборку для построения гибридной сети.

В случае разработки модели ANFIS в виде гибридной сети на основе PID- и IPD-FUZZYрегуляторов показатели ошибки выходного сигнала, ее дифференциала и управляющего воздействия, полученные в результате работы классического регулятора, являются данными, используемыми для проверки эффективности гибридной сети с целью выявления факта ее переобучения, а показатели ошибки выходного сигнала, ее дифференциала и управляющего воздействия, полученные в результате работы нечеткого регулятора, формируют обучающую выборку для построения гибридной сети.

3. Генерирование структуры систем нечеткого вывода типа Сугено, являющихся моделями гибридных сетей.

4. Обучение гибридных сетей в системе MATLAB.

5. Интегрирование сгенерированных систем нечеткого вывода типа Сугено в интерактивную систему Simulink для проведения моделирования.

6. Выполнение исследований работы гибридных моделей в случае следующих изменений ситуаций управления: 
- д для гибридной модели управления техническим объектом с применением PI- и IPIFUZZY-регуляторов вводится возмущающее воздействие, изменяются параметры объекта управления;

- для гибридной модели управления техническим объектом с применением PID- и IPD-FUZZY-регуляторов вводится возмущающее воздействие.

Рассматриваемые в данной работе модели, в которых управление осуществляется с помощью гибридных регуляторов, реализованных на основе последовательного взаимодействия PI- и IPI-FUZZY-регуляторов и PID- и IPD-FUZZY-регуляторов, представлены на рисунках 1 и 2 соответственно.

В схеме моделирования на рисунке 1 и отдельных ее подсистемах участок изменения ситуации управления в виде возмущающего воздействия отмечен пунктирным прямоугольником. Для объекта управления в виде двигателя (ДПТ НВ) изменялись параметры $K_{\text {дв. }}($ с 0,2210 на 0,0128$)$, а также $K_{\text {рег. }}($ с 3,7 на 63,63).

В результате моделирования получены графики переходных процессов и построена поверхность нечеткого вывода (cм. http:// www.swsys.ru/uploaded/image/2021-4/2021-4dop/16.jpg).

В схеме моделирования (регулирование концентрации выходного потока в смесителе) на рисунке 2 участок изменения ситуации управления в виде возмущающего воздействия $(10 \%$ от величины входного воздействия на старте) отмечен пунктирным прямоугольником. В результате моделирования получены графики переходных процессов и построена поверхность нечеткого вывода (см. http://www.swsys.ru/uploaded/image/2021-4/2021-4-dop/17.jpg).

Цифровой анализ качества переходных процессов, выполненный в [10] по основным показателям (величине перерегулирования $(\sigma, \%)$, времени регулирования $\left(t_{\text {рег. }}, \mathrm{c}\right)$, числу колебаний $(n)$, времени достижения первого максимума $\left.\left(t_{1 \max }, \mathrm{c}\right)\right)$, показал, что применение гибридных регуляторов, реализованных на основе последовательного взаимодействия PI- и IPI-FUZZY-регуляторов, а также PID- и IPD-FUZZY-регуляторов со сгенерированной структурой системы нечеткого вывода типа Сугено и разработанной моделью ANFIS и с автоматическим формированием базы правил нечеткого регулятора, полученных с классического регулятора с последующим обучением, позволяет повысить эффективность управления техническим объектом в условиях неполноты данных.

Показатели качества переходных процессов, полученные при управлении объектом с помощью IPI-FUZZY- и IPD-FUZZY-регуляторов, выше, чем при управлении объектом

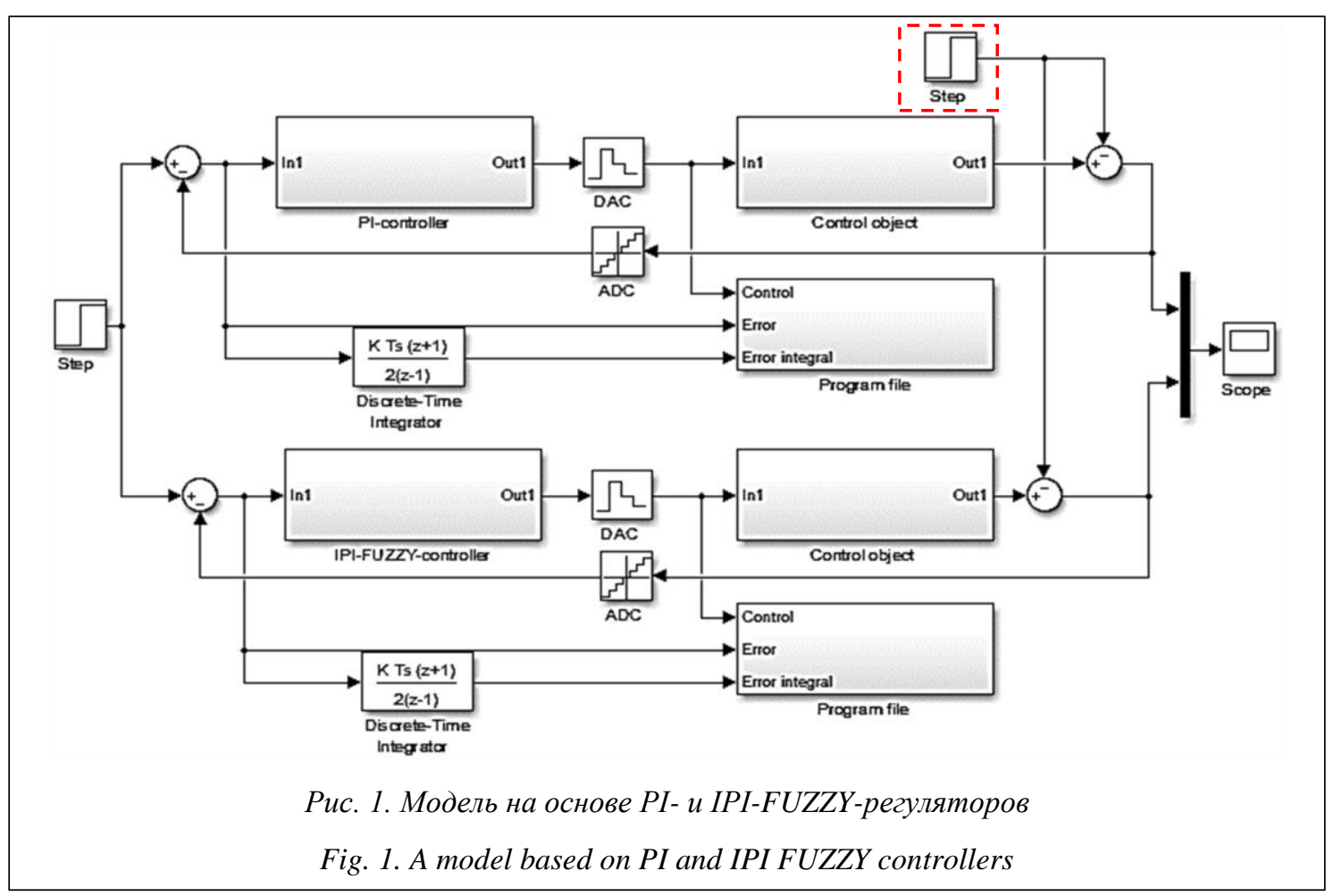




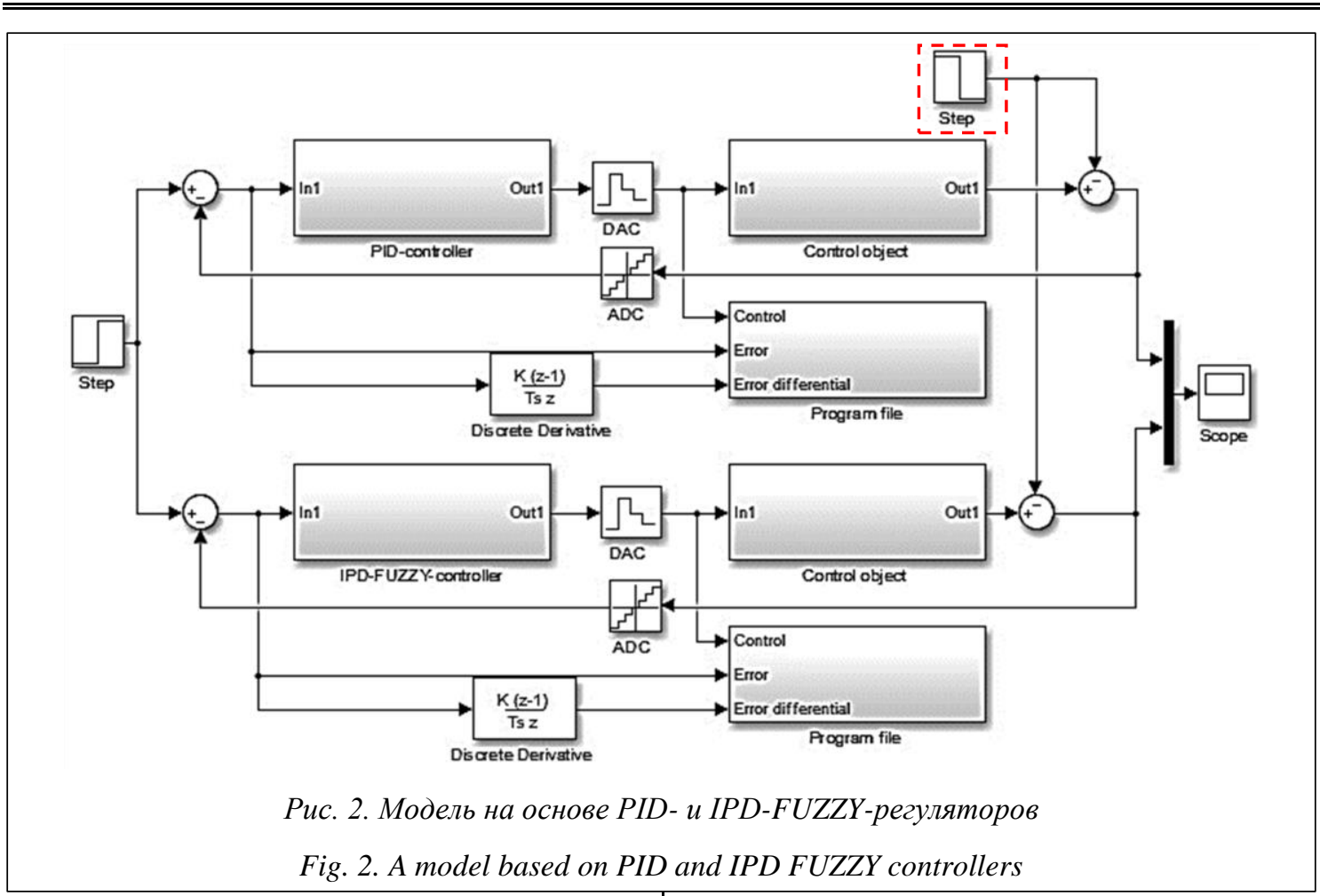

с помощью классических PI- и PID-регуляторов.

Однако в соответствии с поставленной задачей необходимо провести анализ сигналов управления, получаемых в гибридных регуляторах, на основе сравнения результатов работы классического PI-регулятора с IPIFUZZY-регулятором и классического PIDрегулятора с IPD-FUZZY-регулятором для получения информации о возможности применения IPI-FUZZY- и IPD-FUZZY-регуляторов на практике.
Для этого в модели на рисунке 1 получены графики по каждому сигналу (управляющее воздействие, ошибка, интеграл ошибки) после разработки ANFIS в виде гибридной сети на основе PI- и IPI-FUZZY-регуляторов. Графики представлены на рисунке 3.

В модели на рисунке 2 получены графики по каждому сигналу (управляющее воздействие, ошибка, дифференциал ошибки) после разработки ANFIS в виде гибридной сети на основе PID- и IPD-FUZZY-регуляторов. Графики представлены на рисунке 4.

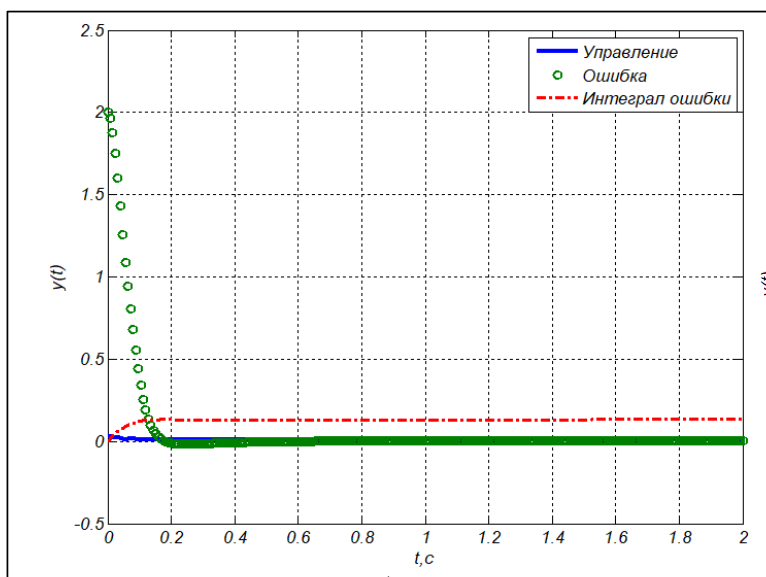

a)

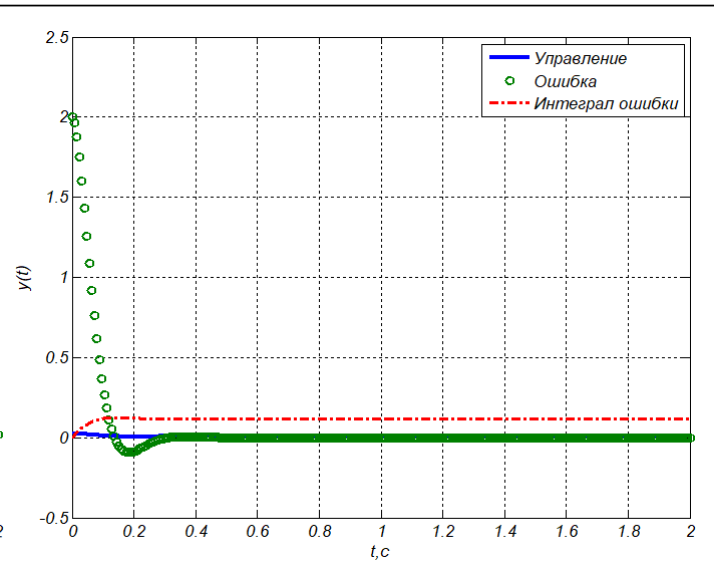

б)

Рис. 3. Графики изменения сигналов управляющего воздействия, ошибки, интеграла ошибки: а) сигналы с PI-регулятора, б) сигналы с IPI- FUZZY-регулятора

Fig. 3. Graphs of changes in the signals of the control action, error, error integral 


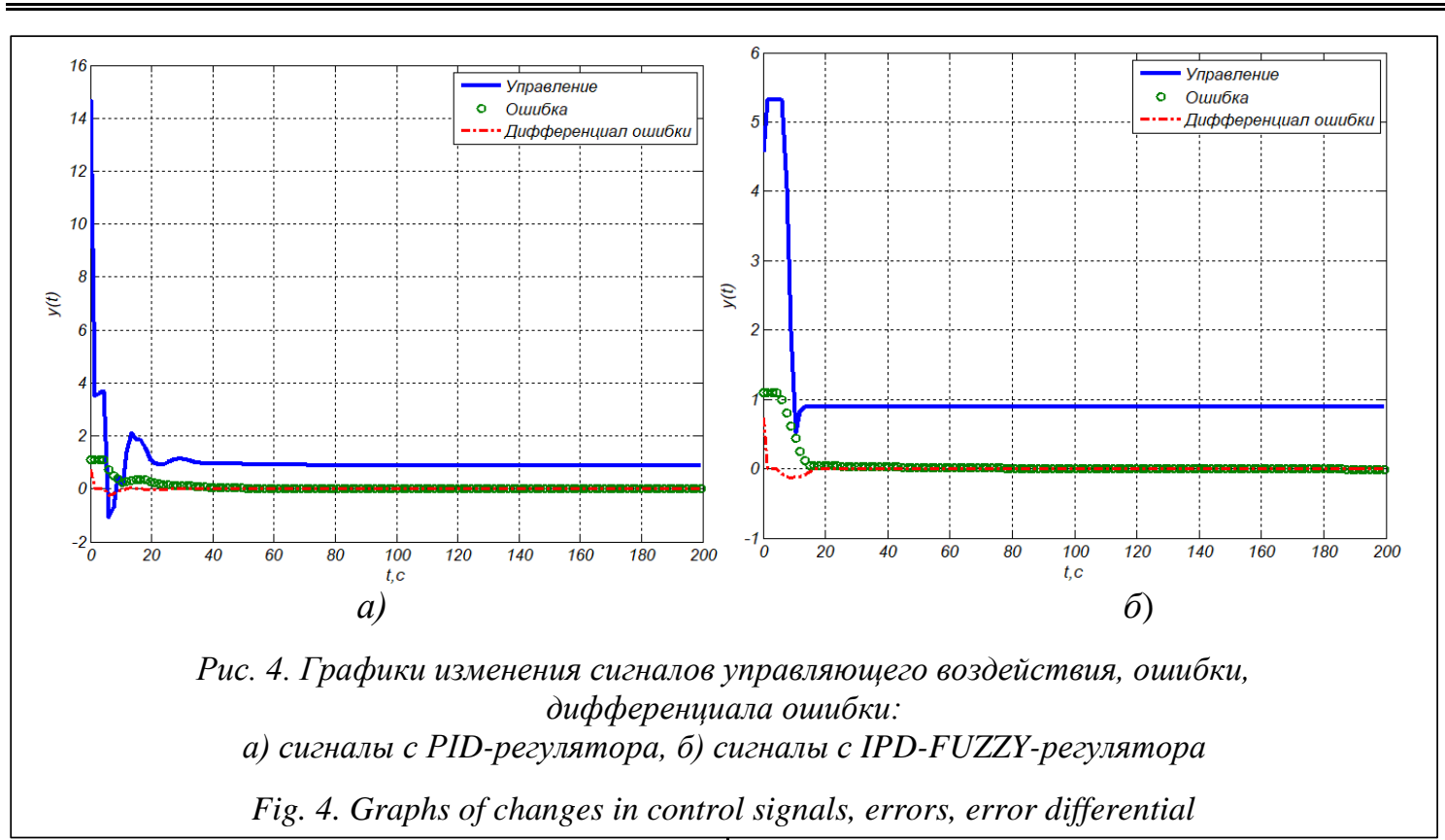

\section{Анализ полученных результатов}

Графики изменения сигналов управляющих воздействий PI- и PID-регуляторов находятся либо на границе отрицательной области, либо в самой области при управлении техническим объектом, функционирующим в изменяющихся условиях. Для объектов управления, входной сигнал у которых может изменяться только в положительных пределах, применение PI- и PID-регуляторов приведет к ухудшению показателей качества. Кроме того, величина перерегулирования с этими регуляторами составила $10 \%$ (рис. 3), что может быть недопустимо по условиям технологического процесса.

Графики изменения сигналов управляющих воздействий IPI-FUZZY - и IPD-FUZZY регуляторов находятся в положительных областях, кроме того, амплитуда управляющих воздействий не превышает 5,2 против 15 у классических регуляторов, что свидетельствует о возможном их применении на практике для получения желаемого управления с максимальным качеством в случае изменения ситуаций управления.

\section{Обсуждение}

Решение современных прикладных задач, связанных с автоматизацией технологических процессов, требует применения концептуально новых подходов и методов управле- ния. Мировое научное сообщество предлагает пути решения таких задач и передовыми традиционными методами, и методами на основе искусственного интеллекта. В большинстве статей, где представлены научные результаты в виде алгоритмов или моделей, проводится моделирование в специальных программных пакетах, позволяющих оценить полученные конечные результаты, например, графики переходных процессов при решении задачи управления техническим объектом.

Однако получение требуемого (удовлетворяющего требованиям) переходного процесса не гарантирует возможность применения той и или иной модели (регулятора) в реальном технологическом процессе. Это показали и результаты представленных в данной статье исследований применительно к управлению техническими объектами с помощью традиционных PI- и PID-регуляторов. При некоторых требованиях к качеству переходных процессов эти PI- и PID-регуляторы не могут быть применены на практике. Поэтому детальный анализ контуров управления в процессе лабораторных исследований необходим для проверки адекватности получаемых результатов.

\section{Заключение}

На реальных производствах АСУ постоянно усложняются, оптимизируются, требуют масштабирования и интеграции. Это 
обусловлено сложностью объектов управления и их количеством. Разработка и программирование реальной АСУ является сложной задачей, зависящей от количества входных и выходных параметров. Еще более сложной является задача разработки научных основ, применяемых в этих системах. Поэтому анализ регуляторов, применяемых в моделях управления техническими объектами, в том числе функционирующими в изменяющихся условиях, представляет научный и практический интерес.

Проведенный в данной статье анализ гибридных регуляторов в моделях управления техническими объектами, функционирующими в изменяющихся условиях, позволил дать адекватную оценку возможности применения представленных регуляторов на практике.

\section{Лumepamypa}

1. Sanchez H.S., Padula F., Visioli A., Vilanova R. Tuning rules for robust FOPID controllers based on multi-objective optimization with FOPDT models. ISA Transactions, 2017, vol. 66, pp. 344-361. DOI: 10.1016/j.isatra.2016.09.021.

2. Savran A., Kahraman G. A fuzzy model based adaptive PID controller design for nonlinear and uncertain processes. ISA Transactions, 2014, vol. 53, no. 2, pp. 280-288. DOI: 10.1016/j.isatra.2013. 09.020 .

3. Hu Y., Yang Y., Li S., Zhou Y. Fuzzy controller design of micro-unmanned helicopter relying on improved genetic optimization algorithm. Aerospace Science and Technology, 2020, vol. 98, art. 105685. DOI: 10.1016/j.ast.2020.105685.

4. Manenti F., Rossi F., Goryunov A.G., Dyadik V.F., Kozin K.A., Nadezhdin I.S., Mikhalevich S.S. Fuzzy adaptive control system of a non-stationary plant with closed-loop passive identifier. Resource-Efficient Technologies, 2015, vol. 1, no. 1, pp. 10-18. DOI: 10.1016/j.reffit.2015.07.001.

5. Kudinov Y.I., Kolesnikov V.A., Pashchenko F.F., Pashchenko A.F., Papic L. Optimization of fuzzy PID controller's parameters. Procedia Computer Science, 2017, vol. 103, pp. 618-622. DOI: 10.1016/ j.procs.2017.01.086.

6. Zeng W., Jiang Q., Xie J., Yu T. A fuzzy-PID composite controller for core power control of liquid molten salt reactor. Annals of Nuclear Energy, 2020, vol. 139, art. 107234. DOI: 10.1016/j.anucene.2019. 107234.

7. Alsafadi L.A., Chulin N.A., Mironova I.V. Synthesis of fuzzy controller based on Simple PID controller. Procedia Computer Science, 2019, vol. 150, pp. 28-38. DOI: 10.1016/j.procs.2019.02.008.

8. Calvo O. Control of power system using adaptive fuzzy controller. IFAC Proceedings Volumes, 2000, vol. 33, no. 6, pp. 69-73. DOI: 10.1016/S1474-6670(17)35450-2.

9. Demidova G.L., Lukichev D.V., Kuzin A.Yu. A genetic approach for auto-tuning of adaptive fuzzy PID control of a telescope's tracking system. Procedia Computer Science, 2019, vol. 150, pp. 495-502. DOI: 10.1016/j.procs.2019.02.084.

10. Игнатьев В.В. Методы управления техническими объектами с помощью интеллектуальных регуляторов на основе самоорганизации баз знаний. Ростов-на-Дону-Таганрог: Изд-во ЮФУ, 2020. 142 c. DOI: $10.18522 / 801273622$.

11. Игнатьев В.В., Спиридонов О.Б., Соловьев В.В., Шаповалов И.О., Игнатьева А.С. Метод оптимального управления техническим объектом, функционирующим в изменяющихся условиях с помощью интеллектуальных регуляторов // Тр. Междунар. науч.-технич. конгресса ИС\&ИТ. 2019. C. 232-247.

12. Игнатьев В.В., Курейчик В.М., Спиридонов О.Б. Моделирование гибридного управления на основе алгоритма самоорганизации робастных баз знаний // Тр. Междунар. науч.-технич. конгресса ИС\&ИТ. 2020. С. 328-336.

13. Ignatyev V., Soloviev V., Beloglazov D., Kureychik V., Ignatyeva A., Vorotova A. System for automatic adjustment of intelligent controller parameters. Proc. Conf. CIT\&DS, 2019, vol. 1084, pp. 226-242. DOI: 10.1007/978-3-030-29750-3_18.

14. Игнатьев В.В. Алгоритм самоорганизации робастных баз знаний без привлечения эксперта в системах управления с интеллектуальными регуляторами // Тр. Междунар. науч.-технич. конгресса ИС\&ИТ. 2020. С. 362-377. 


\title{
Analysis of hybrid controllers in control models of technical objects operating in changing conditions
}

\author{
V.V. Ignatev ${ }^{1}$, Ph.D. (Engineering), Leading Researcher, vvignatev@sfedu.ru \\ V.V. Solovev ${ }^{1}$, Senior Lecturer \\ D.A. Beloglazov ${ }^{1}$, Ph.D. (Engineering), Associate Professor
}

${ }^{1}$ Southern Federal University, Taganrog, 347922, Russian Federation

\begin{abstract}
The paper analyzes hybrid controllers for control models of technical objects operating in changing conditions. It also considers the models which involve control based on hybrid controllers implemented on the basis of sequential interaction between PI- and IPI-FUZZY-controllers and PID- and IPDFUZZY-controllers with the generated structure of the Sugeno-type fuzzy inference system and the developed ANFIS model.

In hybrid controllers, the fuzzy controller rule base is formed automatically using a specially developed algorithm based on data obtained from a classical controller with subsequent training using a neural network. The ANFIS design principle in the form of a hybrid network for PI and IPI-FUZZY controllers is the use of the output signal error indicators, its integral (differential for PID and IPD-FUZZY controllers) and control action. The following aspects have become the development features. In order to test the hybrid network efficiency to identify the fact of its retraining, the authors used the data obtained as a result of the classical regulator operation; to form a training sample for building a hybrid network they used the data obtained as a result of the fuzzy regulator operation. This makes it possible to exclude expert's participation in the synthesis of the fuzzy controller rule base and to ensure efficient and robust control of an object functioning in unforeseen external situations.

The IPI-FUZZY-controller and the IPD-FUZZY-controller have shown better quality indicators comparing to the corresponding classical ones, which makes it possible to recommend using in real control systems. The presented models were developed in the Simulink environment and the ANFIS editor of the Fuzzy Logic Toolbox extension package.
\end{abstract}

Keywords: control, hybrid model, intelligent controller, rule base, learning, uncertainty.

\section{References}

1. Sanchez H.S., Padula F., Visioli A., Vilanova R. Tuning rules for robust FOPID controllers based on multi-objective optimization with FOPDT models. ISA Transactions, 2017, vol. 66, pp. 344-361. DOI: 10.1016/j.isatra.2016.09.021.

2. Savran A., Kahraman G. A fuzzy model based adaptive PID controller design for nonlinear and uncertain processes. ISA Transactions, 2014, vol. 53, no. 2, pp. 280-288. DOI: 10.1016/j.isatra.2013. 09.020 .

3. Hu Y., Yang Y., Li S., Zhou Y. Fuzzy controller design of micro-unmanned helicopter relying on improved genetic optimization algorithm. Aerospace Science and Technology, 2020, vol. 98, art. 105685. DOI: 10.1016/j.ast.2020.105685.

4. Manenti F., Rossi F., Goryunov A.G., Dyadik V.F., Kozin K.A., Nadezhdin I.S., Mikhalevich S.S. Fuzzy adaptive control system of a non-stationary plant with closed-loop passive identifier. Resource-Efficient Technologies, 2015, vol. 1, no. 1, pp. 10-18. DOI: 10.1016/j.reffit.2015.07.001.

5. Kudinov Y.I., Kolesnikov V.A., Pashchenko F.F., Pashchenko A.F., Papic L. Optimization of fuzzy PID controller's parameters. Procedia Computer Science, 2017, vol. 103, pp. 618-622. DOI: 10.1016/ j.procs.2017.01.086.

6. Zeng W., Jiang Q., Xie J., Yu T. A fuzzy-PID composite controller for core power control of liquid molten salt reactor. Annals of Nuclear Energy, 2020, vol. 139, art. 107234. DOI: 10.1016/j.anucene.2019. 107234

7. Alsafadi L.A., Chulin N.A., Mironova I.V. Synthesis of fuzzy controller based on simple PID controller. Procedia Computer Science, 2019, vol. 150, pp. 28-38. DOI: 10.1016/j.procs.2019.02.008.

8. Calvo O. Control of power system using adaptive fuzzy controller. IFAC Proceedings Volumes, 2000, vol. 33, no. 6, pp. 69-73. DOI: 10.1016/S1474-6670(17)35450-2. 
9. Demidova G.L., Lukichev D.V., Kuzin A.Yu. A genetic approach for auto-tuning of adaptive fuzzy PID control of a telescope's tracking system. Procedia Computer Science, 2019, vol. 150, pp. 495-502. DOI: 10.1016/j.procs.2019.02.084.

10. Ignatyev V.V. Methods for Controlling Technical Objects Using Intelligent Controllers Based on Self-Organization of Knowledge Bases. Rostov-on-Don-Taganrog, 2020, 142 p. DOI: 10.18522/ 801273622 (in Russ.).

11. Ignatev V.V., Spiridonov O.B., Solovev V.V., Shapovalov I.O., Ignateva A.S. Optimal control method for a technical object operating under changing conditions using intelligent controllers. Proc. Int. Sci. Tech. Congress IS\&IT, 2019, pp. 232-247 (in Russ.).

12. Ignatev V.V., Kureychik V.M., Spiridonov O.B. Modeling hybrid control based on the self-organization algorithm of robust knowledge bases. Proc. Int. Sci. Tech. Congress IS\&IT, 2020, pp. 328-336 (in Russ.).

13. Ignatyev V., Soloviev V., Beloglazov D., Kureychik V., Ignatyeva A., Vorotova A. System for automatic adjustment of intelligent controller parameters. Proc. Conf. CIT\&DS, 2019, vol. 1084, pp. 226-242. DOI: 10.1007/978-3-030-29750-3_18.

14. Ignatev V.V. A self-organization algorithm for robust knowledge bases without involving an expert in control systems with intelligent controllers. Proc. Int. Sci. Tech. Congress IS\&IT, 2020, pp. 362-377 (in Russ.).

\section{Для цитирования}

Игнатьев В.В., Соловьев В.В., Белоглазов Д.А. Анализ гибридных регуляторов в моделях управления техническими объектами в изменяющихся условиях // Программные продукты и системы. 2021. Т. 34. № 4. С. 555-563. DOI: 10.15827/0236-235X.136.555-563.

\section{For citation}

Ignatev V.V., Solovev V.V., Beloglazov D.A. Analysis of hybrid controllers in control models of technical objects operating in changing conditions. Software \& Systems, 2021, vol. 34, no. 4, pp. 555-563 (in Russ.). DOI: 10.15827/0236-235X.136.555-563. 